\title{
Editorial
}

\section{Anesthetic Management of Neurosurgery Patients in COVID-19 Pandemic in an Emergency Setting}

\author{
Bharath Srinivasaiah ${ }^{1}$ Harsh Deora ${ }^{2, \odot}$ \\ ${ }^{1}$ Department of Neuro-anesthesia and Neuro Critical Care, \\ National Institute of Mental Health and Neurosciences, Bangalore, \\ Karnataka, India \\ 2Department of Neurosurgery, National Institute of Mental Health \\ and Neurosciences, Bangalore, Karnataka, India
}

J Neurosci Rural Pract 2020;11:514-516

We read with interest the institutional protocol for anesthetic management of a patient undergoing neurosurgery during the COVID-19 pandemic. We concur with this protocol, which is similar to our hospital's protocol, except for a few modifications appropriate for our institute. Perhaps this relates to conducting multiple neurosurgical emergency surgeries daily. When community transmission becomes widespread, every patient needs to be considered as a suspect. Anesthesiologists form a key group of healthcare workers who are susceptible to the COVID-19 infection, owing to airway management during surgical interventions and management of critically ill patients. Modifications in airway management are required to minimize aerosol generation. The protocols should have clear workflow guidelines which will help in facilitating multidisciplinary communication to implement effective isolation practices. ${ }^{1}$ Ensuring training of healthcare workers regarding donning and doffing of personal protective equipment (PPE) and conducting simulation exercises are essential to ensure compliance to protocol, especially in emergencies like management of neurotrauma. ${ }^{2}$ We believe the protocol is well made and may benefit from some modifications in institutions dealing with neurosurgical emergencies and trauma.

\section{Preanesthetic Consultation (PAC)}

Face masks have been shown to reduce the infectious load and chance of contagion; hence, it would be advisable that every patient must wear a face mask during the PAC. ${ }^{3}$ Meticulous airway assessment after donning PPE is a must to avoid unanticipated difficult airway and help formulate airway plan. Screening tools and subsequent COVID-19 testing of a suspected patient have to be modified for each institution based on the progression of disease in the community to reduce transmission of infection. Awake craniotomy patients present a unique challenge where careful selection of patients and appropriate preoperative counselling is essential. Discussion with neurosurgeon if the case is high-risk in nature, regarding the urgency of operation and delay, if possible, or about measures which may mitigate the emergency in the form of an external ventricular drain (EVD); coiling for aneurysms and antiedema measures might prove beneficial. ${ }^{4-6}$

\section{High-Risk Procedures for Aerosolization}

High-risk surgical procedures would include, in addition to those mentioned, transnasal pituitary procedures, skull base surgeries, CUSA for decompression, tracheostomy, and any other surgery where aerosol generation is possible, like drilling or ultrasonic aspirator use. ${ }^{4}$ Perhaps the use of low-speed electric drills, copious irrigation, and avoidance of a breach of sinus would be advisable. ${ }^{5}$ High-risk anesthetic procedures would be intubation, extubation, mask ventilation, open suctioning of respiratory secretions, awake intubation, highflow nasal oxygen therapy, and cardiopulmonary resuscitation. Low-risk procedures also include EVD placement and lumbar drain placement.

\section{Preparation of the Operation Theater}

Formulation of an early plan and having an intubation checklist prevents unnecessary delays and errors. If a negative pressure room is not available, ensure ventilation with 15 to 20 air exchanges per hour, with the direction of airflow

\author{
Address for correspondence \\ Harsh Deora, MCh, DNB, Assistant \\ Professor, Department of \\ Neurosurgery, National Institute of \\ mental health and Neurosciences, \\ Bangalore, Karnataka, India \\ (e-mail: demo5601@gmail.com).
}

DOI https://doi.org/ $10.1055 / \mathrm{s}-0040-1713342$ ISSN 0976-3147. C2020 Association for Helping
Neurosurgical Sick People

\section{License terms}

$($ () (1) $\Theta \circledast$ 
such that fresh air enters near the health worker and exhaust air is removed close to the patient. Establishing a scavenging system is recommended to not allow the ventilated gases to enter the room air. Only necessary equipment is to be kept, and the use of disposable ones is recommended in confirmed cases. Clear delineation of roles, airway plan, and crossmonitoring of all team members for potential contamination, along with reducing the number of individuals in the theater at a time, would be beneficial.

Regional guidelines, as per country or institutional protocol for PPE, should be maintained.

\section{Reusing N95 Mask}

Efforts must be made to ensure fit testing of the N95 mask for all at-risk staff, as nonfit-tested use of N95 mask contributed to transmission to healthcare workers. Alternatives to N95 mask such as half-/full-face respirators, or powered air-purifying respirators, can be used if available. These have shown good efficacy and better protection.

\section{Transfer of Patient into an Operation Theater (OT)}

Use of airborne infection isolation room (AIIR) for intubation when available and then wheeling in the patient into OT also reduces the risk of transmission. It is recommended to have an anteroom to prevent infectious particles from escaping the OT into the corridors. ${ }^{7}$

\section{Anesthetic Management}

Use the lowest possible gas flows for preoxygenation and give fentanyl slowly to reduce coughing. Paralyze early with rocuronium to avoid manual ventilation, However, if manual ventilation is needed, then apply small tidal volumes only. Ensure full neuromuscular blockade to avoid coughing during intubation using train-of-four (TOF) monitoring. Use video laryngoscopic technique which confers prompt and reliable intubation. Do not use techniques you are not familiar with. Confirm tube position by observing bilateral chest rise/ultrasound/ capnography, as auscultation will be difficult and risk contamination due to PPE. Secure endotracheal tube to avoid displacement during positioning-supine/ lateral/prone/sitting, especially in neurosurgical patients, and avoid disconnections during positioning. Check for endobronchial migration of tube with flexion of neck, if needed for surgery so as to avoid ventilator disconnections later. In neurosurgical patients, the use of supraglottic airway devices is limited, except in unanticipated difficult airway. It is recommended to use the second generation supraglottic airway devices. Conduct of awake intubation is not recommended. However, if inevitable, then take every precaution to minimize aerosol generation. ${ }^{8}$

Lower cranial nervemonitoring electrode placement should be done after intubation, and in case of hypotension, have vasopressor ready. Avoid circuit disconnection-push twist all connections. Ensure normocapnia with lung-protective mechanical ventilation (MV) strategy (low-tidal volume and high rate) to reduce the chances of brain bulge. Deep clean the room 20 minutes after tracheal intubation. After extubating, ensure that patient wears a facemask immediately and strictly adheres to doffing protocols. Ensure hand hygiene and organize a team briefing after the surgery. Discard all disposable sensors used for neuromonitoring., ${ }^{9,10}$

\section{Pediatric Neurosurgical Patients}

We need to consider preprocedural sedation to reduce anxiety, increase compliance for IV (intravenous) line placement, or $^{7}$ nasal premedication is undesirable due to the risk of sneezing. Also avoid parental presence for induction to reduce exposure. IV induction is preferred, and inhalational induction needs to be avoided. However, crying while awake IV line placement has a higher risk of exposure. Rapid sequence induction is recommended if appropriate. To avoid hypoxemia during intubation, one may deliver gentle positive pressure ventilation to achieve chest rise while maintaining a tight mask seal. Using a cuffed endotracheal tube, to reduce leak, is advised. If uncuffed ET is used, then use the appropriate size to minimize leak. ${ }^{11,12}$

\section{Modifications in CPR}

Perhaps considering apneic ventilation instead of bag and mask ventilation would be beneficial. Intubation should be done early and inflate the cuff to isolate the airway. Hold chest compressions during the intubation attempt. Consider using an automated chest compression device if available. ${ }^{13,14}$

The protocol described by the authors is complete and comprehensive and thus underlines our preparedness during this unprecedented global emergency. Safe practice during this period can effectively "flatten the curve" of both COVID-19 cases and the surge of neurosurgical emergencies.

\section{Funding \\ None. \\ Conflict of Interest \\ None declared.}

\section{References}

1 Nedunchezhian AS, Ajayan N, P APH, Prathapadas U, Sethuraman M, Koshy T. Finding the calm in the chaos: An institutional protocol for anesthetic management of a patient for neurosurgery during coronavirus disease 2019 pandemic. J Neurosci Rural Pract 2020;11(3):369-374

2 Salazar LRM, Agrawal D, Deora H, Agrawal A. Neurotrauma in the time of SARS-COV 2: a checklist for its safe management. J Neurosci Rural Pract 2020;11(3):474-477

3 Soto DM, Cardona Maya WD, Londoño E, Bueno Sánchez JC. The feasibility of generalized face mask usage during COVID-19 pandemic: a perspective from Latin America. Infect Control Hosp Epidemiol 2020. Doi: 10.1017/ice.2020.227

4 Gupta P, Muthukumar N, Rajshekhar V, et al. Neurosurgery and neurology practices during the novel COVID-19 Pandemic: a consensus statement from India. Neurol India 2020 68(2):246-254 
5 Lo YT, Yang Teo NW, Ang BT. Editorial. Endonasal neurosurgery during the COVID-19 pandemic: the Singapore perspective. J Neurosurg 2020. Doi:10.3171/2020.4.JNS201036

6 Deora H, Kim SH, Behari S, et al; World Federation of Neurosurgical Societies (WFNS) Spine Committee. Anterior surgical techniques for cervical spondylotic myelopathy: WFNS spine committee recommendations. Neurospine 2019; 16(3):408-420

7 Matava CT, Kovatsis PG, Summers JL, et al; PeDI-Collaborative. Pediatric airway management in COVID-19 patientsConsensus Guidelines from the Society for Pediatric Anesthesia's Pediatric Difficult Intubation Collaborative and the Canadian Pediatric Anesthesia Society. Anesth Analg 2020. Doi: $10.1213 /$ E.0000000000004872

8 Malhotra N, Joshi M, Datta R, Bajwa SJ, Mehdiratta L. Indian Society of Anaesthesiologists (ISA National) Advisory and Position Statement regarding COVID-19. Indian J Anaesth 2020;64(4):259-263

9 Greenland JR, Michelow MD, Wang L, London MJ. COVID-19 infection: implications for perioperative and critical care physicians. Anesthesiology 2020;132(6):1346-1361

10 World Health Organization. (2020). Rational use of personal protective equipment (PPE) for coronavirus disease (COVID-19): interim guidance, 19 March 2020. World Health Organization. Available at: https://apps.who.int/iris/handle/ $10665 / 331498$
11 Wong J, Goh QY, Tan Z, et al. Preparing for a COVID-19 pandemic: a review of operating room outbreak response measures in a large tertiary hospital in Singapore. Se préparer pour la pandémie de COVID-19: revue des moyens déployés dans un bloc opératoire d'un grand hôpital tertiaire au Singapour. Can J Anaesth. 2020;67(6):732-745

12 Cook TM, El-Boghdadly K, McGuire B, McNarry AF, Patel A, Higgs $A$, Consensus guidelines for managing the airway in patients with COVID-19: Guidelines from the Difficult Airway Society, the Association of Anaesthetists the Intensive Care Society, the Faculty of Intensive Care Medicine and the Royal College of Anaesthetists. Anaesthesia. 2020;75(6):785-799.

13 Meng L, Qiu H, Wan L., et al. Intubation and Ventilation amid the COVID-19 Outbreak: Wuhan's Experience. Anesthesiology. 2020;132(6):1317-1332

14 Tang G, Kam A. Perioperative management of suspected/ confirmed cases of COVID- 19. ATOTW 421:2020. Available at: https://www.wfsahq.org/components/com_virtual_library/ media/1c4ec5c64b9aaacf7c47f76a61fb6edc-atow-422-01.pdf. Accessed May 25, 2020 